\title{
Impacto de dois programas de educação nutricional sobre o risco cardiovascular em pacientes hipertensos e com excesso de peso ${ }^{1}$
}

\author{
Impact of two nutritional education programs \\ on cardiovascular risk in overweight \\ hypertensive patients
}

Tatiana Souza ALVAREZ2

Maria Teresa ZANELLA²

\section{RE S U M O}

\section{Objetivo}

Comparar os efeitos de dois protocolos de um programa de intervenção nutricional sob o risco cardiovascular em pacientes hipertensos e com excesso de peso em uso de medicamentos usual. Os pacientes foram acompanhados no Ambulatório de Hipertensão e Metabologia Cardiovascular, Universidade Federal de São Paulo.

\section{Métodos}

A população do estudo foi constituída por 63 pacientes hipertensos e com excesso de peso [índice de massa corporal $\geq 27$ e $\left.<39 \mathrm{~kg} / \mathrm{m}^{2}\right]$, com a média de idade de $M=55,7, D P=6,1$ anos; 12 homens e 51 mulheres, divididos em dois grupos de acordo com a freqüência das visitas, a cada cinco semanas ( $G 35 \mathrm{n}=25$ ) ou a cada duas semanas ( G14 $n=38$ ), durante 20 semanas. Os dois grupos foram submetidos a um programa de intervenção nutricional que englobou atendimentos em grupos, nos quais foram abordados conceitos de alimentação saudável. No início e no término do período do estudo, todos os pacientes foram submetidos a medidas de pressão arterial, avaliação antropométrica e avaliação laboratorial para cálculo do Risco Coronariano de Framingham.

\section{Resultados}

Ao término do estudo, foi observada redução no índice de massa corporal, na circunferência da cintura $(\mathrm{M}=102,9, \mathrm{DP}=9,7 \mathrm{~cm}$ vs $\mathrm{M}=101,8, \mathrm{DP}=7,6 \mathrm{~cm} \mathrm{NS})$, na pressão arterial sistólica $(\mathrm{M}=139,7, \mathrm{DP}=12,4 \mathrm{mmHg}$ vs $M=134,8, D P=8,2 \mathrm{mmHg} N S)$, no colesterol total sérico $(M=192,9, D P=35,7 \mathrm{mg} / \mathrm{dL}$ vs $\mathrm{M}=195,2, \mathrm{DP}=37,8 \mathrm{mg} / \mathrm{dL}$

\footnotetext{
$\overline{1}$ Artigo elaborado a partir da tese de T.S. ALVAREZ, intitulada "Impacto de um programa de educação nutricional sobre o risco cardiovascular em pacientes hipertensos e com excesso de peso". Universidade Federal de São Paulo; 2007.

2 Universidade Federal de São Paulo, Hospital do Rim e Hipertensão, Ambulatório de Hipertensão e Metabologia Cardiovascular. R. Leandro Dupret, 365, Vila Clementino, 04025-011, São Paulo, SP, Brasil. Correspondência para/Correspondence to: T.S ALVAREZ. E-mail: <tatiana.alvarez@terra.com.br>.
} 
NS), no consumo energético referido ( $M=1759,5, D P=385,8 \mathrm{Kcal}$ vs $M=1858,7, D P=452,8 \mathrm{kcak}$ NS) e no RCF $(\mathrm{M}=11,1, \mathrm{DP}=6,1$ vs $\mathrm{M}=9,4, \mathrm{DP}=3,5 \mathrm{NS})$.

\section{Conclusão}

A intervenção nutricional educativa de curto prazo, se mostrou eficaz para redução do risco cardiovascular em pacientes hipertensos e com excesso de peso. Em relação a freqüência das visitas, pôde-se verificar que a visita mensal já produz resultados positivos no sentido de modificar os hábitos alimentares e reduzir o risco cardiovascular.

Termos de indexação: Doenças cardiovasculares. Educação nutricional. Fatores de risco. Hipertensão.

\section{A B S T R A C T}

\section{Objective}

To evaluate and compare the effects of two study protocols with a program of educational nutrition intervention on CARDIOVACULAR risk in overweight hypertensive patients maintained on their usual medication. Patients were followed at Hypertension and Metabology Division of Federal University of São Paulo.

\section{Methods}

Sixty tree overweight [body mass index $\geq 27$ e $<39 \mathrm{~kg} / \mathrm{m}^{2}$ ] hypertensive patients, age $M=55,7$ (Standard deviation - $S D=6,1)$ years old, 12 men and 51 women, were divided into two groups according to visit intervals, either every five weeks (G35 n=25) or every two weeks (G14n=38), during twenty weeks. The two groups were submitted to an educational program aiming to improve the quality of their diet and to promote changes in their alimentary habits. Before and at the end of the study period, all patients were submitted to blood pressure determinations, to anthopometric measurements and to laboratorial evaluation for the calculation of Framingham coronary risk.

\section{Results}

At the end of the study significant and similar changes in body mass index, waist circumference (102,9, $S D=9,7$ vs $101,8, S D=7,6$ NS), systolic blood pressure $(139,7, S D=12,4$ vs $134,8, S D=8,2$ NS), total cholesterol $(192,9, S D=35,7$ vs 195,2, SD=37,8 NS), energy intake (1759,5, SD=385, 8 vs 1858,7, SD=452,8 NS) and Framingham coronary risk $(11,1, S D=6,1$ vs 9,4, SD=3,5 NS) we observed in G14 and G35 groups respectively.

\section{Conclusion}

A short program of nutritional intervention was shown to be useful to reduce coronary risk in overweight hypertensive patients despite a very small change in body weight. A higher frequency of visits did not achieve better results, with a monthly visit being enough for positive changes although with a higher rate of drop outs.

Indexing terms: Cardiovascular disease. Nutrition education. Factors risk. Hypertension.

\section{N T R O D U ÇÃ O}

Diversos estudos epidemiológicos têm fornecido conhecimentos sobre os fatores envolvidos na etiologia das doenças cardiovasculares. Entre os fatores de risco cardiovascular destacam-se: hipertensão arterial, dislipidemias, obesidade, diabetes mellitus e algumas condições relacionadas ao estilo de vida inadequado, como: tabagismo, inatividade física e hábitos alimentares desfavoráveis (alto consumo de carboidratos simples e gorduras, além de baixo consumo de fibras) ${ }^{1}$.
O impacto das modificações dos hábitos alimentares na redução do risco cardiovascular tem sido avaliado em diversos estudos e como resultados têm sido observadas a diminuição do peso corporal, melhora da tolerância à glicose, a redução da pressão arterial e a melhora do perfil lipídico do plasma.

O estudo Interhearth realizado com 252 centros de 52 países dos 5 continentes incluiu 27098 pacientes com idade média de 56 anos, que foram avaliados quanto ao estilo de vida, 
incluindo fatores psicossociais, consumo de frutas e vegetais, consumo regular de álcool, tabagismo, atividade física e presença de fatores de risco cardiovascular. Os objetivos do estudo foram testar a associação desses fatores com a ocorrência de infarto do miocárdio. A respeito do consumo de frutas e vegetais, o risco de infarto do miocárdio foi $30 \%$ menor nos indivíduos com consumo regular, comparado ao grupo sem consumo diário. Nos indivíduos que além do consumo regular de frutas e vegetais exerciam atividade física regular e não possuíam o hábito de fumar o risco de infarto do miocárdio foi $80 \%$ menor $^{2}$.

Discute-se os resultados das intervenções objetivando mudanças de hábitos dependem da freqüência de atendimentos, do tipo de atendimento, ou de ambos, individualmente ou em grupo.

No estudo realizado por Melin et al. ${ }^{3}, 43$ pacientes obesos que também apresentavam outras comorbidades tais como diabetes, hipertensão, ovário policístico e apnéia do sono, foram divididos em dois grupos que receberam orientação dietética e orientação para modificação do estilo de vida, de forma mais ou menos intensiva. O tratamento mais intensivo incluía visitas a cada quatro semanas no primeiro ano e bimestralmente no segundo ano, enquanto o tratamento menos intensivo incluía atendimentos trimestrais. Os resultados demonstraram, após dois anos, que a perda de peso foi muito semelhante entre os dois grupos assim como a adesão ao tratamento e a freqüência de desistências.

Outro estudo realizado por Hakala et al. ${ }^{4}$ avaliou por 5 anos 60 pacientes com obesidade que foram divididos em dois grupos destinados a receber atendimento individual ou em grupo por 2 anos. Ambos os atendimentos compreendiam orientações sobre estratégias para redução do peso que incluíam orientação dietética, incentivo à atividade física regular e modificação de comportamento alimentar. O programa menos intensivo consistia de atendimentos individuais mensais no primeiro ano e depois a cada quatro meses, no segundo ano. O outro mais intensivo consistia de um período de internação de duas semanas durante as quais os pacientes foram atendidos em grupo em um total de 40 horas. Posteriormente os atendimentos ocorreram semanalmente durante 10 meses e após mensalmente até se completarem 2 anos. Os pacientes tratados em grupo apresentaram uma perda de peso mais rápida nas duas semanas em que permaneceram internados, enquanto que melhores resultados em dois anos foram observados no atendimento individual, particularmente em homens. Ao final de cinco anos grande parte do peso perdido foi recuperado nos dois grupos que se mostraram semelhantes quanto à manutenção do peso perdido. Esses estudos sugerem que vários fatores, além da freqüência de atendimento durante determinado período de tempo e do tipo de atendimento, interferem na aquisição permanente de um estilo de vida saudável. Apesar disto, a educação alimentar se mostra importante como estratégia a ser adotada em Saúde Pública para conter o avanço da obesidade, principal determinante das condições que contribuem para o maior risco cardiovascular. A mudança do comportamento alimentar pela conscientização é o ponto fundamental para uma modificação nutricional mais eficiente.

Propostas educativas em nutrição com atendimentos em grupos objetivam tornar o ambiente interativo e facilitador de aprendizagem. Por meio do trabalho em grupo é possível observar uma crítica reflexiva dos pacientes, com a possibilidade de mudança de hábitos, proporcionando assim melhor qualidade de vida ${ }^{5}$. Na área de saúde, pelo fato de os tratamentos individuais se mostrarem dispendiosos, tem sido dada preferência aos atendimentos em grupo.

Os objetivos do presente estudo foram avaliar e comparar dois esquemas de intervenção nutricional educativa sobre os fatores de risco cardiovascular em pacientes obesos e hipertensos, em tratamento medicamentoso para as diversas co-morbidades.

\section{MÉTODOS}

O protocolo deste estudo foi aprovado pelo Comitê de Ética em Pesquisa e este trabalho segue 
os princípios éticos contidos na Declaração de Helsinki, além de atender às legislações específicas para pesquisas realizadas no Brasil.

A população do estudo foi constituída de pacientes hipertensos (pressão arterial sistólica maior ou igual a 140mmHg, pressão arterial diastólica maior ou igual a $90 \mathrm{mmHg}$ ou em uso de medicação anti-hipertensiva), obesos ou com excesso de peso (índice de massa corporal de 27 a $39,9 \mathrm{~kg} / \mathrm{m}^{2}$ ) com faixa etária de 45 a 70 anos que possuíssem, no mínimo, mais um dos clássicos fatores de risco cardiovascular ou que tivessem sofrido um acidente vascular prévio (infarto do miocárdio ou acidente vascular cerebral). Foram considerados fatores de risco cardiovascular: diabetes mellitus, dislipidemias, tabagismo e sedentarismo.

Os pacientes selecionados foram divididos em dois grupos, destinados a atendimentos periódicos a cada 14 dias (grupo G14) ou a cada 35 dias (grupo G35) por um total de 140 dias. Assim o Grupo G14 compareceu a 10 visitas e o grupo G35 compareceu a 4 visitas.

Fizeram parte deste estudo 92 pacientes, divididos aleatoriamente, por meio de sorteio, de acordo com a freqüência de atendimento, em dois grupos, cada um contendo 46 pacientes. No primeiro atendimento nove pacientes desistiram de participar, sendo três pacientes do G14 e seis pacientes do G35. Cinco pacientes do G14 e 15 pacientes do G35 não retornaram para segunda visita. Assim, contou-se com 38 pacientes no G14 e 25 pacientes no G35.

No início e ao término do período do estudo foram realizadas medidas de pressão arterial, antropometria, avaliação laboratorial para cálculo do risco coronariano de Framingham e avaliação do consumo alimentar. A avaliação antropométrica consistiu da medida da massa corporal, estatura, circunferências da cintura e do quadril para obtenção dos índices de massa corporal (IMC) e relação cintura-quadril, respectivamente. A avaliação laboratorial foi feita a partir de dosagens de glicemia de jejum, hemoglobina glicada, triglicérides, colesterol total e frações: LDL-col e HDL-col, em amostras sangüíneas colhidas após jejum de 12 horas. O cálculo do risco coronariano de Framingham foi realizado para verificar o risco do paciente apresentar um evento coronariano em um período de 10 anos $^{6}$.

Para analisar os hábitos alimentares dos pacientes foi utilizado o registro alimentar de três dias, antes e após orientação nutricional. Para avaliar a quantidade de alimento consumido, as informações obtidas foram convertidas em gramas e calculadas pelo software NutWin ${ }^{7}$. Quanto à qualidade da dieta foi analisada a variedade de alimentos a partir dos grupos alimentares contidos na pirâmide alimentar brasileira ${ }^{8}$. Os pacientes não foram orientados a seguir uma dieta com conteúdo energético pré-determinado e restrita em energia. Durante as visitas foram oferecidas orientações nutricionais de forma interativa permitindo a participação dos pacientes, com atividades em grupo para que eles mesmos elaborassem a própria dieta a partir dos conhecimentos adquiridos. Como recursos visuais foram utilizados: Iousa e retroprojetor. A elaboração do conteúdo programático foi baseada nas seguintes referências: Ministério da Saúde ${ }^{9}$, Cervatto ${ }^{10}$ e Boog ${ }^{11}$. Nas visitas foram aplicados os seguintes recursos pedagógicos-nutricionais: roda dos alimentos, interpretação de rótulos, refeição fora de casa e abordagem sobre influência da mídia na alimentação.

Para a análise dos resultados foi utilizado o software estatístico Statistical Package for Social Science (SPSS) versão 10.0 for Windows.

Os resultados foram analisados utilizando-se da estatística descritiva, tendo-se calculado média (M) e desvio-padrão (DP). Recorreu-se à estatística inferencial por meio do teste $t$ de Student pareado, para comparação entre pré e pós-teste. Para comparação entre os grupos utilizou-se o teste $t$ de Student para amostras independentes. O nível mínimo de significância estabelecido foi de $p<0,05$.

\section{RE S U L T A D O S}

Os pacientes estudados possuíam a média de idade de $M=56,7, D P=5,6$ anos para $\mathrm{G} 14$ e 
$M=54,1, D P=6,6$ anos para $G 35$, sem diferença estatisticamente significante entre os grupos. Em relação ao sexo, observou-se predominância no sexo feminino (81,6\% - G14 e 80\% - G35). Quanto ao nível de escolaridade, grande parte dos pacientes referira possuir o ensino fundamental (G14 - 72\% e G35 - 76,3\%).

Quanto à dieta, no início do estudo o valor energético médio referido no registro alimentar no grupo G14 foi de 1759,5 quilocalorias e no grupo G35 foi de 1858,7 quilocalorias, uma redução significante do consumo energético registrado ocorreu em ambos os grupos, como demonstrado na Tabela 1. Apesar disto ao final do estudo avaliando-se a distribuição de macronutrientes em relação ao valor energético total, os dois grupos comportaram-se de maneira semelhante. Foram observadas reduções similares no conteúdo de carboidratos nos dois grupos, embora no G14 esta redução tenha sido marginalmente significante $(p=0,059)$. Da mesma maneira foram observados aumentos semelhantes no consumo de proteínas, embora no G35 este aumento tenha se mostrado também marginalmente significante $(p=0,059)$. Houve aumento no consumo de fibras no G14, mas não no $\mathrm{G} 35$ enquanto o consumo de colesterol reduziu igualmente nos dois grupos.
Quanto à variedade dos alimentos, verificou-se que a redução de carboidratos na dieta nos dois grupos deve-se à redução no consumo de açúcares, cereais, pães, raízes e tubérculos. 0 aumento do consumo de proteínas no G14, provavelmente, ocorreu pela maior ingestão de leguminosas enquanto que no $\mathrm{G} 35$ foi devido à maior ingestão de produtos lácteos. Nos dois grupos houve aumento no consumo de hortaliças e redução no consumo de óleos e gorduras, como demonstrado na Tabela 2.

Os dados de avaliação antropométrica e da pressão arterial, ao início e final do estudo, estão apresentados na Tabela 3. Os dois grupos analisados não diferiram entre si com relação ao índice de massa corporal e às circunferências. O IMC e a circunferência da cintura apresentaram redução significante em ambos os grupos e as variações observadas $(\Delta)$ não diferiram entre eles. A medida da circunferência do quadril apresentou redução significante somente no G14.

Quanto à pressão arterial na condição basal, os dois grupos não diferiram quanto aos valores da sistólica e diastólica. Houve redução com $p<0,05$ na pressão diastólica em ambos os grupos, enquanto que a sistólica reduziu somente no G14.

Tabela 1. Distribuição das médias e desvios-padrão referentes a variações dietéticas de pacientes hipertensos, baseadas no registro alimentar, antes e após intervenção. São Paulo (SP), 2007.

\begin{tabular}{|c|c|c|c|c|c|c|c|c|c|c|c|c|}
\hline \multirow{3}{*}{ Nutrientes } & \multicolumn{6}{|c|}{ G14 } & \multicolumn{6}{|c|}{ G35 } \\
\hline & \multicolumn{2}{|c|}{ Pré-teste } & \multicolumn{2}{|c|}{ Pós-teste } & \multicolumn{2}{|c|}{$\Delta \mathrm{G} 14$} & \multicolumn{2}{|c|}{ Pré-teste } & \multicolumn{2}{|c|}{ Pós-teste } & \multicolumn{2}{|c|}{$\Delta \mathrm{G} 35$} \\
\hline & M & $\mathrm{DP}$ & M & $\mathrm{DP}$ & M & $\mathrm{DP}$ & M & $\mathrm{DP}$ & M & DP & M & $\mathrm{DP}$ \\
\hline Valor calórico (kcal) & 1759,5 & 385,8 & 1480,5 & $191,9 *$ & $-279,0$ & 415,6 & 1858,7 & 452,8 & 1420,9 & $194,1^{*}$ & $-437,8$ & 503,5 \\
\hline Carboidratos (\%) & 49,1 & 7,9 & 46,1 & 6,7 & $-3,0$ & 9,4 & 50,7 & 8,6 & 47,7 & $6,6^{*}$ & $-3,0$ & 7,1 \\
\hline Proteínas (\%) & 18,1 & 4,3 & 20,8 & $6,2^{*}$ & 2,7 & 6,0 & 17,0 & 4,6 & 19,5 & 5,6 & 2,5 & 6,9 \\
\hline Lípides (\%) & 32,8 & 8,0 & 33,1 & 4,9 & 0,3 & 9,2 & 32,4 & 7,9 & 32,9 & 6,2 & 0,5 & 8,2 \\
\hline AGS (\%) & 7,9 & 2,8 & 7,6 & 2,1 & $-0,3$ & 3,8 & 7,5 & 2,4 & 7,4 & 2,1 & $-0,1$ & 3,1 \\
\hline MUFA (\%) & 10,0 & 3,1 & 10,4 & 2,3 & 0,4 & 3,7 & 9,9 & 3,0 & 10,4 & 2,8 & 0,4 & 3,6 \\
\hline PUFA (\%) & 11,8 & 3,5 & 12,6 & 2,2 & 0,8 & 3,7 & 12,3 & 4,1 & 12,7 & 2,3 & 0,4 & 3,8 \\
\hline Fibras(g) & 16,9 & 7,3 & 19,8 & $4,8^{*}$ & 2,9 & 7,9 & 19,4 & 11,3 & 21,1 & 9,9 & 1,7 & 8,9 \\
\hline Sódio (mg) & 2059,6 & 735,3 & 2150,2 & 783,6 & $90,6 \pm$ & 988,9 & 2126,4 & 723,6 & 1983,4 & 637,4 & $-143,0$ & 1082,3 \\
\hline Potássio (mg) & 2575,8 & 1239,3 & 2315,0 & 514,1 & 260,8 & 1294,4 & 2398,2 & 888,0 & 2267,0 & 749,5 & $-131,2$ & 1127,2 \\
\hline Colesterol (mg) & 555,2 & 246,2 & 186,4 & $95,3^{*}$ & $-368,8$ & 277,2 & 531,9 & 187,9 & 205,4 & 126,0 * & $-326,5$ & 223,3 \\
\hline
\end{tabular}

* $p<0,05$.

AGS: ácido graxo saturado, MUFA: ácido graxo monoiinsaturado, PUFA: ácido graxo poliinsaturado; M: média, DP: desvio-padrão, G14: acompanhamento nutricional a cada duas semanas, G35: acompanhamento nutricional a cada cinco semanas. 
Tabela 2. Caracterização do consumo alimentar dos pacientes atendidos segundo a variedade da alimentação, estudo por número de porções, antes e após orientação. São Paulo (SP), 2007.

\begin{tabular}{|c|c|c|c|c|c|c|c|c|c|c|c|c|}
\hline \multirow{3}{*}{ Variáveis } & \multicolumn{6}{|c|}{ G14 } & \multicolumn{6}{|c|}{ G35 } \\
\hline & \multicolumn{2}{|c|}{ Pré-teste } & \multicolumn{2}{|c|}{ Pós-teste } & \multicolumn{2}{|c|}{$\Delta \mathrm{G} 14$} & \multicolumn{2}{|c|}{ Pré-teste } & \multicolumn{2}{|c|}{ Pós-teste } & \multicolumn{2}{|c|}{$\Delta \mathrm{G} 35$} \\
\hline & M & DP & M & DP & M & DP & M & DP & $\mathrm{M}$ & DP & M & DP \\
\hline Cereais, pães, raízes e tubérculos & 5,7 & 0,9 & 4,3 & $0,7^{\star}$ & $-1,4$ & 1,1 & 5,8 & 1,2 & 4,2 & $0,7^{*}$ & $-1,7$ & 1,3 \\
\hline Hortaliças & 2,3 & 1,1 & 3,2 & $0,6^{*}$ & 0,9 & 1,2 & 1,8 & 0,9 & 3,1 & $0,7^{*}$ & 1,3 & 1,1 \\
\hline Frutas & 2,0 & 1,4 & 2,4 & 0,9 & 0,4 & 1,7 & 2,4 & 1,9 & 2,3 & 0,7 & $-0,1$ & 2,3 \\
\hline Carnes, aves, peixe e ovos & 1,7 & 0,8 & 1,8 & 0,5 & 0,1 & 0,8 & 1,9 & 0,9 & 1,8 & 0,4 & $-0,2$ & 1,0 \\
\hline Produtos lácteos & 1,7 & 0,9 & 1,7 & 0,5 & 0,1 & 0,9 & 1,6 & 0,9 & 2,0 & $0,5^{*}$ & 0,4 & 0,8 \\
\hline Leguminosas & 1,0 & 0,7 & 1,3 & $0,4^{*}$ & 0,3 & 0,7 & 1,1 & 0,8 & 1,2 & 0,3 & 0,2 & 0,9 \\
\hline Açúcares & 1,2 & 1,0 & 0,5 & $0,6^{*}$ & $-0,7$ & 1,0 & 1,5 & 1,6 & 0,5 & $0,9^{*}$ & $-0,9$ & 1,2 \\
\hline Óleos e gorduras & 1,9 & 0,9 & 1,2 & $0,2^{*}$ & $-0,6$ & 0,9 & 2,1 & 1,0 & 1,3 & $0,4^{*}$ & $-0,7$ & 0,9 \\
\hline
\end{tabular}

* $p<0,05$.

G14: acompanhamento nutricional a cada duas semanas; G35: acompanhamento nutricional a cada cinco semanas; M: média; DP: desvio-padrão.

Tabela 3. Distribuição dos grupos de pacientes hipertensos, segundo avaliação antropométrica e pressão arterial, antes e após intervenção. São Paulo (SP), 2007.

\begin{tabular}{|c|c|c|c|c|c|c|c|c|c|c|c|c|}
\hline \multirow{3}{*}{ Variáveis } & \multicolumn{6}{|c|}{ G14 } & \multicolumn{6}{|c|}{ G35 } \\
\hline & \multicolumn{2}{|c|}{ Pré-teste } & \multicolumn{2}{|c|}{ Pós-teste } & \multicolumn{2}{|c|}{$\Delta \mathrm{G} 14$} & \multicolumn{2}{|c|}{ Pré-teste } & \multicolumn{2}{|c|}{ Pós-teste } & \multicolumn{2}{|c|}{$\Delta \mathrm{G} 35$} \\
\hline & M & $\mathrm{DP}$ & $M$ & $\mathrm{DP}$ & $M$ & DP & M & $\mathrm{DP}$ & M & DP & M & DP \\
\hline IMC (kg/m²) & 33,3 & 3,6 & 32,5 & $3,4^{*}$ & $-0,8$ & 0,8 & 32,9 & 4,0 & 32,3 & $4,0^{*}$ & $-0,6$ & 0,3 \\
\hline Circunferência da cintura $(\mathrm{cm})$ & 102,9 & 9,7 & 101,4 & $9,8^{*}$ & $-1,5$ & 3,9 & 101,8 & 7,6 & 100,5 & $7,7^{*}$ & $-1,3$ & 2,3 \\
\hline Circunferência do quadril (cm) & 112,4 & 9,3 & 111,0 & $9,0^{*}$ & $-1,4$ & 4,1 & 109,8 & 8,4 & 108,8 & 8,2 & $-1,0$ & 2,7 \\
\hline Relação cintura/quadril & 0,9 & 0,1 & 0,9 & 0,1 & 0,0 & 0,0 & 0,9 & 0,7 & 0,9 & 0,7 & 0,0 & 0,0 \\
\hline Pressão arterial sistólica $(\mathrm{mmHg})$ & 139,7 & 12,4 & 136,2 & $9,5^{\star}$ & $-3,6$ & 8,8 & 134,8 & 8,2 & 133,2 & 6,3 & $-1,6$ & 4,7 \\
\hline Pressão arterial diastólica (mmHg) & 88,8 & 7,9 & 84,7 & $5,7^{*}$ & $-4,1$ & 6,6 & 85,8 & 6,4 & 83,6 & $5,7^{*}$ & $-2,2$ & 5,0 \\
\hline
\end{tabular}

* $p<0,05$.

G14: acompanhamento nutricional a cada duas semanas; G35: acompanhamento nutricional a cada cinco semanas; M: média; DP: desvio-padrão.

Tabela 4. Comparação dos exames laboratoriais e risco coronariano de Framingham de grupos de pacientes hipertensos. São Paulo (SP), 2007.

\begin{tabular}{|c|c|c|c|c|c|c|c|c|c|c|c|c|}
\hline \multirow{3}{*}{ Exames laboratoriais } & \multicolumn{6}{|c|}{ G14 } & \multicolumn{6}{|c|}{ G35 } \\
\hline & \multicolumn{2}{|c|}{ Pré-teste } & \multicolumn{2}{|c|}{ Pós-teste } & \multicolumn{2}{|c|}{$\Delta \mathrm{G} 14$} & \multicolumn{2}{|c|}{ Pré-teste } & \multicolumn{2}{|c|}{ Pós-teste } & \multicolumn{2}{|c|}{$\Delta \mathrm{G} 35$} \\
\hline & M & DP & M & DP & M & DP & M & $\mathrm{DP}$ & $\mathrm{M}$ & DP & M & $\mathrm{DP}$ \\
\hline Glicemia (mg/dL) & 118,0 & 31,4 & 109,5 & 29,9 & $-8,5$ & 26,4 & 135,6 & 51,0 & 118,6 & 48,0 & $-17,1$ & 52,6 \\
\hline Hemoglobina glicada (\%) & 6,6 & 1,1 & 6,6 & 1,2 & $-0,2$ & 1,8 & 7,3 & 1,4 & 6,9 & 1,3 & $-0,2$ & 1,0 \\
\hline Triglicérides (mmol/L) & 157,8 & 69,9 & 135,1 & $64,5^{*}$ & $-4,4$ & 36,4 & 192,1 & 120,9 & 173,9 & 152,1 & $-14,5$ & 31,0 \\
\hline Colesterol total $(\mathrm{mmol} / \mathrm{L})$ & 192,9 & 35,7 & 188,5 & 40,3 & $-22,7$ & 51,7 & 195,2 & 37,8 & 180,6 & $35,9^{*}$ & 18,2 & 91,9 \\
\hline $\mathrm{HDL}(\mathrm{mmol} / \mathrm{L})$ & 53,8 & 15,6 & 55,4 & 15,2 & 1,6 & 9,0 & 49,7 & 16,2 & 53,1 & 17,3 & 3,4 & 12,2 \\
\hline $\mathrm{LDL}(\mathrm{mmol} / \mathrm{L})$ & 105,7 & 33,7 & 106,4 & 36,0 & 0,7 & 35,2 & 112,0 & 31,2 & 92,9 & $26,7^{*}$ & $-17,6$ & $29,5 \#$ \\
\hline Risco em 10 anos (\%) & 11,1 & 6,1 & 8,9 & $4,6^{*}$ & $-2,2$ & 3,8 & 9,4 & 3,5 & 7,6 & $3,9^{*}$ & $-1,8$ & 3,8 \\
\hline
\end{tabular}

* $p<0,05$.

G14: acompanhamento nutricional a cada duas semanas; G35: acompanhamento nutricional a cada cinco semanas; M: média; DP: desvio-padrão. 
Com relação ao perfil metabólico observaram-se reduções nos níveis séricos de triglicérides apenas no G14 e nos níveis de colesterol total somente no $\mathrm{G} 35$, como apresentado na Tabela 4. O risco coronariano avaliado pelo escore de Framingham mostrou redução semeIhante e significante em ambos os grupos, como também descrito na Tabela 4.

\section{I S C U S S Ã O}

Os pacientes incluídos neste estudo e submetidos à intervenção nutricional educativa e a estímulos para mudanças no estilo de vida, possuíam hábitos alimentares não recomendáveis. Eram sedentários, com excesso de peso e apresentavam fatores de risco cardiovascular. Tratava-se de uma amostra de pacientes na maturidade, com hábitos muito arraigados e, portanto com menor chance de conseguir alterar de forma permanente o estilo de vida. Neste estudo procurou-se, a partir de dois esquemas de visitas periódicas, transmitir aos pacientes informações e ensinamentos que pudessem melhorar a qualidade da dieta sem, no entanto, restringir diretamente o consumo de energia, objetivando a melhora da condição de risco cardiovascular. O objetivo era, portanto, o de aumentar o conhecimento dos pacientes quanto ao valor energético e nutricional dos alimentos e eliminar hábitos inadequados para que eles mesmos pudessem estabelecer uma dieta mais apropriada às próprias condições de saúde.

Os resultados do presente estudo mostram alteração nos valores do IMC, da circunferência de cintura e no perfil metabólico que resultaram em reduções significativas e semelhantes, quanto ao risco coronariano de Framingham, próximas a $20 \%$, nos dois grupos. Em estudo realizado por Pugliese $^{12}$, que incluía pacientes hipertensos com perfil semelhante ao deste estudo, durante 11 meses, dois grupos foram estudados: um grupo recebia informações sobre os fatores de risco cardiovascular e outro grupo, além de receber estes conhecimentos, era submetido à intervenção psicológica, que visava a identificar e abordar conflitos psicológicos envolvidos nas dificuldades para mudança de estilo de vida. Essa intervenção produziu redução em torno de $27 \%$ no risco de Framingham, enquanto no grupo que apenas recebeu orientação não houve redução do risco. No grupo que recebeu intervenção psicológica, o aumento no HDL-col associou-se à redução de peso, o que leva a crer que esta intervenção tenha feito com que os pacientes aderissem à orientação dietética fornecida pelos médicos.

A perda de peso observada no presente estudo foi considerada pequena, cerca de $2 \%$, comparada a outros trabalhos que referem ser necessária uma perda de peso mínima de 5\% para obtenção de reduções significativas na pressão arterial, nos níveis séricos de lípides, além de melhora na intolerância à glicose e de prevenção do diabetes melittus ${ }^{13,14}$. Neste estudo, apesar da baixa perda de peso, observou-se redução da pressão arterial, dos triglicérides, colesterol total e LDL-col. Isto provavelmente ocorreu devido à melhora na qualidade da dieta, que passou a conter menor quantidade de carboidratos simples e gorduras saturadas e maior quantidade de fibras. De fato, estudos epidemiológicos também relatam que o consumo de frutas, hortaliças, leguminosas e grãos integrais pode auxiliar na redução do risco cardiovascular. A ingestão de fibras induz a redução do colesterol sangüíneo e também diminui a velocidade de esvaziamento gástrico, contribuindo para melhor controle glicêmico em pacientes diabéticos ${ }^{1}$.

No estudo não foi oferecida orientação específica para redução do consumo energético referido no registro alimentar inicial. Apesar disso, os pacientes dos dois grupos apresentaram uma redução no consumo energético diário. Embora o consumo energético referido, tanto no início como no final do estudo, estivesse muito abaixo do gasto energético estimado para estes pacientes e pudesse não corresponder à realidade, houve redução espontânea no consumo de energia, que pode ter contribuído para perda de peso e melhora do perfil metabólico. 
Em relação à ingestão de proteínas, foi observado no grupo G14 um aumento no consumo de leguminosas e no $\mathrm{G} 35$ de produtos lácteos. $\mathrm{O}$ aumento do consumo de leguminosas pode ser considerado positivo, pois são boas fontes de proteínas e fibras, além de serem de baixo custo. $\mathrm{O}$ aumento na ingestão de leite e derivados no G35 indica maior ingestão de cálcio, que foi incentivada em ambos os grupos, uma vez que nesta faixa etária da população estudada é comum ocorrer uma perda óssea de cálcio particularmente nas mulheres na pós menopausa.

Em relação ao consumo de lípides referido nos registros alimentares, houve redução no consumo de colesterol dietético, que pode ter sido a causa da redução dos níveis séricos de colesterol total e LDL-col no grupo G35, embora reduções semelhantes não tenham sido observadas no G14.

Assim, a modificação qualitativa da dieta pode resultar em benefícios em curto prazo em pacientes com alto risco cardiovascular. Nestes pacientes, Belmelmans et al. ${ }^{15}$ demonstraram redução do risco de Framingham em 3\% com acompanhamento durante três anos e por meio de registros alimentares destes pacientes, uma redução do consumo energético por meio da diminuição do consumo de gordura saturada, colesterol e aumento de fibras. Resultados semeIhantes ocorreram neste estudo com maior redução do risco de Framingham em menor tempo de acompanhamento. Isto sugere que ao longo do tempo os pacientes tendem a abandonar os hábitos alimentares recomendados e voltam aos antigos. Intervenções periódicas, portanto, podem ser necessárias para os manter em condições de menor risco cardiovascular. A proposta deste acompanhamento foi realizar uma intervenção nutricional educativa que ampliasse os conhecimentos de nutrição dos pacientes assistidos e não apenas fornecer uma dieta com conteúdo energético e de macronutrientes pré-determinados.

Neste estudo observou-se que o programa de intervenção foi capaz de promover alterações favoráveis no consumo alimentar habitual após cinco meses de acompanhamento que resultaram em perda de peso, redução da pressão arterial e melhora do perfil metabólico. Mesmo que não se possa inferir que as alterações tenham ocorrido estritamente por conta da modificação nutricional educativa e dirigida, os resultados deste estudo revelam uma tendência em favor da modificação da dieta em decorrência desta ação. $O$ aumento dos conhecimentos e a discussão em grupo das si-tuações do cotidiano alimentar possibilitam melhores escolhas, que podem resultar em mudança duradoura no comportamento alimentar, embora avaliação em longo prazo não tenha sido feita.

Neste estudo foram comparados também dois esquemas de reuniões periódicas com freqüências diferentes, para verificar a hipótese de melhores resultados nos pacientes com maior freqüência de atendimentos. Esta hipótese, entretanto, não se confirmou sendo os resultados muito semelhantes em ambos os grupos, embora um maior número de pacientes tenha permanecido no G14 do que no G35. Portanto deve-se questionar a relação custo-benefício de atendimentos quinzenais, podendo estes serem realizados mensalmente, o que propiciaria o atendimento a um maior número de pacientes e reduziria os custos do Sistema Único de Saúde.

\section{ONCLUSÃO}

A intervenção nutricional educativa de curto prazo, com atendimentos a cada cinco semanas, em grupos, por cinco meses, foi eficaz para estimular a mudança qualitativa dos hábitos alimentares e reduzir os fatores de risco cardiovascular. Os dados do estudo sugerem que uma freqüência maior de visitas dentro do programa educativo não resulta em melhores resultados.

\section{COLABORADORES}

T.S. ALVAREZ e M.T. ZANELLA participaram igualmente da coleta de dados, da discussão dos resultados e da elaboração do artigo. 


\section{REFERÊ N CIAS}

1. Farret JF. Nutrição e doenças cardiovasculares: prevenção primária e secundária. São Paulo: Atheneu; 2005.

2. Yosuf S, Hawkewn S, Ônpuu S, Dans T, Avezum A, Lanas $F$, et al. Effect of potentially modifiable risk factors associated with myocardial infarction in 52 countries (the INTERHEARTH study): case-control study. Lancet. 2004; 364(9438):937-952.

3. Melin I, Karlström B, Lappalainen R, Berglund L, Mohsen R, Vessby B. A programme of behaviour modification and nutrition counselling in the treatment of obesity: a randomised 2-y clinical trial. Int J Obes Relat Metab Disord. 2003; 27(9): 1127-1135.

4. Hakala P, Karvetti RL, Ronnemaa T. Group vs. individual weight reduction programmes in the treatment of severe obesity: a five year follow-up study. Int J Obes Relat Metab Disord. 1993;17(2): 97-102.

5. Tchakinakian LA. A alimentação na promoção da saúde em idosos hipertensos: testando uma nova técnica [dissertação]. São Paulo: Pontifícia Universidade Católica de São Paulo; 2002.

6. Hubert HB, Fenileib M, McNamara PM. Obesity as independent risk factor for cardiovascular disease: a 267-year follow-up of the participants in the Framingham Hearth Study. Circulation. 1983; 67(5): 968-77.

7. NutWin. Programa de Apoio à Nutrição [programa de computador]. São Paulo: Departamento de Informática da Universidade Federal de São Paulo; 199-.
8. Phillipi ST, Latterza AR, Cruz ATR, Ribeiro LC. Pirâmide alimentar adaptada: guia para escolha dos alimentos. Rev Nutr. 1999; 12(1):65-80.

9. Brasil. Ministério da Saúde. Secretaria de Políticas de Saúde. Educação nutricional para alunos do ensino fundamental. Brasília; 2001.

10. Cervatto AM. Intervenção nutricional educativa: promovendo a saúde de adultos e idosos em universidade abertas à terceira idade [tese]. São Paulo: Universidade de São Paulo; 1999.

11. Boog MCF, Motta DG. Educação nutricional. São Paulo: IBRASA; 1984.

12. Pugliese R. Avaliação da eficácia de uma intervenção psicológica destinada à redução do risco coronariano em pacientes hipertensos [tese]. São Paulo: Universidade Federal de São Paulo; 2005.

13. Willett WC, Dietz WH, Colditz GA. Guidelines for health weight. N Eng J Med. 1999; 341(6): 427-34.

14. Sociedade Brasileira de Cardiologia. V Diretrizes Brasileiras de Hipertensão Arterial. São Paulo: SBC; 2006.

15. Bemelmans WJE, Broer J, Hulshof KFAM, Siero FW, May JF, Meyboom-de-Jong B. Long-term effects of nutritional group education for persons at high cardiovascular risk. Eur J Public Health. 2004; 14(3):240-5.

Recebido em: 16/4/2008

Versão final reapresentada em: 21/7/2008 Aprovado em: 5/11/2008 
